\title{
PAPER
}

\section{Alterations in brain activation during cholinergic enhancement with rivastigmine in Alzheimer's disease}

\author{
S A R B Rombouts, F Barkhof, C S van Meel, P Scheltens
}

J Neurol Neurosurg Psychiatry 2002;73:665-671

See end of article for authors' affiliations

......................

Correspondence to: Dr S A R B Rombouts, Dept KFI, Vrije Universiteit

Medical Centre, PO Box 7057, $1007 \mathrm{MB}$

Amsterdam, Netherlands; sarb.rombouts@vumc.nl

Received

27 February 2002

In revised form 2 July 2002

Accepted 15 August 2002

\begin{abstract}
Background: Rivastigmine enhances cholinergic activity and has been shown in clinical trials to decrease the rate of deterioration in Alzheimer's disease. It remains unclear where in the brain it exerts its effect. Functional magnetic resonance imaging (fMRI) can be used to measure changes in brain function and relate these to cognition.

Objectives: To use fMRI to study brain activation with rivastigmine treatment.

Methods: The effect on brain activation of a single dose of rivastigmine was tested in seven patients with mild Alzheimer's disease using fMRI during face encoding, and in five patients during a parametric working memory task.

Results: During face encoding, rivastigmine increased bilateral activation in the fusiform gyrus. Brain activation was also enhanced in the prefrontal cortex in a simple working memory task. When working memory load was further increased, not only was increased activation seen, but in certain areas there was also decreased activation.

Conclusions: These findings link the previously observed increase in cognitive performance in Alzheimer's disease after treatment with a cholinesterase inhibitor to altered brain activation. Although the results cannot be generalised to the Alzheimer's disease population at large, they provide evidence that in mild Alzheimer's disease, rivastigmine enhances brain activation in the fusiform and frontal cortices. This is compatible with the concept of cholinergic circuitry.
\end{abstract}

O ne of the critical features of Alzheimer's disease is degeneration of cholinergic neurones, located in the basal forebrain nuclei, progressively depriving the brain of its cholinergic input. This reduces alertness, short term memory, and learning abilities. Current treatment strategies in Alzheimer's disease are aimed at replacing or enhancing cholinergic activity. Rivastigmine is a drug that enhances cholinergic activity by inhibiting the enzyme acetylcholinesterase, which otherwise metabolises the neurotransmitter acetylcholine extremely rapidly.

It has been shown in rats that rivastigmine particularly inhibits acetylcholinesterase in the neocortex and hippocampus, the brain regions most affected in Alzheimer's disease. ${ }^{12}$ In clinical trials, patients with mild to moderate Alzheimer's disease experienced significantly less deterioration in cognitive indices and were found to show improvement in activities of daily living. ${ }^{1-3}$ However, these data in humans do not link brain function and behaviour-that is, it remains unclear where in the brain rivastigmine exerts its effect on brain function.

Functional magnetic resonance imaging (fMRI) can be used to measure changes in brain function and relate these to cognition. In this pilot study we use fMRI to test the effect of a single dose of rivastigmine on memory related brain activation in patients with mild Alzheimer's disease in two memory systems: episodic memory and working memory. The first functional system of interest was assessed by a face encoding task, the second by a working memory task.

\section{METHODS}

\section{Patients and drugs}

Eleven patients with Alzheimer's disease from the memory outpatient clinic of the Vrije Universiteit Medical Centre/ Alzheimer Centre were asked to participate. Written informed consent for the fMRI procedure was obtained. The clinical diagnosis of probable Alzheimer's disease was made by a senior neurologist according to the NINCDS-ADRDA criteria
(National Institute of Neurological and Communicative Disorders and Stroke - Alzheimer's Disease and Related Disorders Association). ${ }^{4}$ Disease severity was assessed according to the clinical dementia rating (CDR) scale, ${ }^{5}$ while cognitive status was expressed independently of the CDR rating by the mini-mental state examination (MMSE) scores. ${ }^{6}$

Each patient was studied in two separate sessions, with a seven day period in between. Half of the patients were drug-free at the first session, and received a single $3 \mathrm{mg}$ dose of rivastigmine three hours before scanning for the second session. The other half received treatment in the reverse order. The investigators were blinded to the drug administration schemes. In each scanning session, patients were questioned for side effects, such as mild dizziness and nausea, both before and after scanning.

\section{Data acquisition}

Imaging was performed on a $1.5 \mathrm{~T}$ magnetic resonance unit with a gradient echo, echo planar imaging (EPI) sequence with $3 \mathrm{~mm}$ in plane resolution, a slice thickness of $5 \mathrm{~mm}$ (1 $\mathrm{mm}$ gap), a repetition time of $2.48 \mathrm{~s}$, and an echo time of 60 ms. From within the bore of the scanner, patients looked through a mirror mounted on the head coil to a back projection screen on which stimuli were projected. In their right hand, patients held an MRI compatible response box (Lightwave Medical Industries). For each stimulus, a response with the right hand was requested by pressing a key with either the index finger (left button) or the middle finger (right button).

Abbreviations: $C D R$, clinical dementia rating; $E P I$, echo planar imaging; fMRI, functional magnetic resonance imaging; MMSE, mini-mental state examination; $\mathrm{R}+/-$, with/without rivastigmine; SPM, statistical parametric mapping 


\section{Cognitive tasks}

Two weeks before scanning, patients received a paper version of the two tasks, to familiarise themselves with the test procedure. On the day of scanning, both tasks were again explained in detail and practiced on a computer outside the scanner. Patients also practiced the tasks inside the scanner. When necessary, patients wore MRI compatible glasses to correct visual acuity.

Task 1 was a face encoding task, based on previous studies on face encoding. ${ }^{7-9}$ Thirty two faces were presented in four blocks of eight faces ( 40 seconds per block). Blocks with faces alternated with blocks presenting grey rectangles (eight stimuli per block, 40 seconds per block). Patients were instructed to remember the faces and knew that they would be tested for face recognition afterwards. To make sure patients attended to the stimuli, they had to indicate whether the faces were male or female (left or right button), or whether the projected grey rectangles were dark or light grey (left or right button), indicated by two large arrows at the bottom of the screen. In all, 144 scans were obtained. After completion of task 2 (see below), face recognition was tested (no fMRI data acquired) using a two choice forced recognition in which 16 items were presented, each consisting of two faces (one seen, one unseen). Test scores were the percentage of correct answers.

Task 2 was a parametric n-back non-spatial working memory task. ${ }^{10}$ The task consisted of four different conditions, randomly presented in blocks: instruction, fixation, a "target $=X$ " condition (referred to as $X$ ), a "l-back" condition (which we will refer to as "simple working memory"), and a "2-back" condition (corresponding to "increased working memory load"). Each of the conditions started with an instruction that was presented visually for 10 seconds. Letters were then presented sequentially: every two seconds one letter was presented ( 1.5 seconds for presentation, followed by black screen for 0.5 second). In the FIX condition, patients simply had to fixate on a cross projected on the middle of the screen disappearing and reappearing at the same rate as the letters. In the $X$ condition, patients had to press the left button with their index finger when the letter $\mathrm{X}$ appeared on the screen (hit rate: 1 in 5 ). In the simple working memory condition, patients had to press the button when a letter was repeated without any other letter intervening (for example A ...A). In the increased working memory load condition, patients had to press the button when a letter was repeated with one other letter in between (for example A ...B ...A). In the working memory conditions, the hit rate was also 1 in 5 . Conditions were randomly repeated in 12 different blocks (20 letters per block, block duration 40 seconds), and 245 scans were obtained in all in one session. Reaction times were recorded and test scores were determined by subtracting the false positive from the true positive rate.

\section{Data analysis}

Data were analysed by statistical parametric mapping using a fixed effects design (SPM99, Wellcome Department of Cognitive Neurology, London, UK). ${ }^{12}$ All EPI volumes (session 1 and 2 , task 1 and 2) were realigned to the first volume of the face encoding task of experiment 1 , after removing the first five volumes of each task to account for induction of a steady state. EPI volumes were then normalised to a standard EPI template volume based on the Montreal Neurological Institute reference brain in the space of Talairach and Tournoux, ${ }^{13}$ resliced using sinc interpolation in space to $3 \times 3 \times 3 \mathrm{~mm}$ voxels, and spatially smoothed with an $8 \mathrm{~mm}$ full width at half maximum Gaussian kernel.

Data were modelled using a box car design, convolved with the haemodynamic response function, and both high pass and low pass frequency filters were applied in the time domain to remove low and high frequency components. For each condi- tion, variables were estimated by multiple linear regression analysis. Specific effects were tested by applying appropriate linear contrasts to the variable estimates for each condition, resulting in a $t$ statistic for every voxel. We applied a corrected probability $(p)$ value of 0.05 based on false discovery rate statistics ${ }^{14}$ with a minimum cluster size of 10 voxels. In task 1 , the effect of interest was signal increase during face encoding. In task 2, there were two effects of interest: (1) signal increases between "1-back" and "target $=X$ " (1-back $>X)$, corresponding to simple working memory; (2) signal increase between "2-back" and "target $=X "$ " (2-back $>X)$, corresponding to increased working memory load. The effects of interest were calculated both in the untreated arm ( $\mathrm{R}-$ ) and in the rivastigmine $\operatorname{arm}(\mathrm{R}+)$. Both positive $(\mathrm{R}+>\mathrm{R}-)$ and negative treatment effects $(\mathrm{R}+<\mathrm{R}-)$ were tested for each of the three contrasts outlined above.

A possible danger in testing treatment effects is the inclusion of regions that show a signal decrease in the memory test when one is not interested in detecting changes in negative activation. To exclude such regions in this study, the contrasts of treatment effects were masked with SPMs of signal decrease with $\mathrm{p}<0.05$ (exclusive masking, uncorrected) as follows: when testing positive effects $(\mathrm{R}+>\mathrm{R}-)$, the SPM of signal decrease in R- was used for masking (that is, voxels with a signal decrease in $\mathrm{R}$ - were excluded in the analysis). In this way, we can be sure that positive treatment effects can only result from increased positive BOLD signal between $\mathrm{R}+$ and $\mathrm{R}-$, and not from a decrease in negative activation during $\mathrm{R}+$ compared with $\mathrm{R}-$. Following the same reasoning, the negative treatment effect $(\mathrm{R}+<\mathrm{R}-)$ was masked with the SPM of signal decrease in $\mathrm{R}+$

Based on previous studies employing comparable tasks, and given our main interest in episodic and working memory, we limited the analysis for the face encoding task to the medial temporal lobe (episodic memory) and also the ventral occipital cortex (fusiform gyrus, higher visual processing). ${ }^{7-9}$ Analysis of the working memory task was limited to the frontal and parietal lobes. ${ }^{1011}$

\section{RESULTS}

Two patients could not participate in the fMRI procedure because they experienced moderately severe gastrointestinal side effects of rivastigmine (nausea, vomiting). Two other patients had to be excluded because of claustrophobia and neck complaints while lying in the scanner. The other patients did not complain of any side effect. The remaining patients (four male, three female, mean (SD) age 71 (8) years) had an average MMSE score of 24.7 (2.0); all patients had a CDR rating of 1 .

\section{Face encoding}

The remaining seven patients successfully completed the face encoding tasks in the two sessions. Of these, five patients received rivastigmine in the second session and two in the first session. Owing to a computer error, test scores were not recorded in two patients, and reaction times were not recorded in three. Overall, 93\% of male/female decisions were correct during encoding, with no differences between arms; similarly, there were no differences in reaction times (table 1). During face recognition after scanning, patients performed just above chance and again with no difference between arms.

In both arms ( $\mathrm{R}-$ and $\mathrm{R}+$ ), bilateral activation was found along the ventral route in the fusiform gyrus, but no activation in the medial temporal lobe (table 2 ). The contrast $\mathrm{R}+>\mathrm{R}-$ showed enhanced activation in the left and right fusiform gyrus with rivastigmine (fig 1, table 2). Detailed inspection of the activation maps showed that the local maximum of the contrast $\mathrm{R}+>\mathrm{R}-$ in the right fusiform gyrus was activated in both $\mathrm{R}-$ and $\mathrm{R}+$ (that is, activation already present in $\mathrm{R}-$ was 
Table 1 Test scores and reaction times of the face encoding and working memory task in the rivastigmine arm $(R+)$ and the rivastigmine-free arm $(R-)$

\begin{tabular}{|c|c|c|c|c|c|c|}
\hline & \multicolumn{3}{|c|}{ Rivastigmine $(R+)$} & \multicolumn{3}{|c|}{ No rivastigmine ( $\mathrm{R}-$ ) } \\
\hline \multicolumn{7}{|l|}{ Face encoding } \\
\hline Test score $(\%)(n=5)$ & $92(2)$ & & & $94(6)$ & & \\
\hline Reaction time $(s)(n=4)$ & $1.17(0.16)$ & & & $1.09(0.08)$ & & \\
\hline \multicolumn{7}{|l|}{ Working memory task } \\
\hline & $x$ & 1-back & 2-back & $x$ & 1-back & 2-back \\
\hline Test score $(n=5)$ & $0.98(0.04)$ & $0.84(0.17)$ & $0.67(0.32)$ & $0.95(0.06)$ & $0.93(0.11)$ & $0.75(0.34)$ \\
\hline Reaction time $(s)(n=5)$ & 0.64 (0.09) & $0.71(0.09)$ & $0.90(0.04)$ & $0.61(0.06)$ & $0.65(0.05)$ & $0.88(0.05)$ \\
\hline
\end{tabular}

Table 2 Talairach coordinates of local maxima and sizes of activated clusters in the face encoding task in the rivastigmine-free arm, the rivastigmine arm, and the comparison of these two

\begin{tabular}{lllllll}
\hline & \multicolumn{2}{l}{ Talairach coordinates } & & \\
\cline { 2 - 4 } & $\mathrm{x}$ & $\mathrm{y}$ & $\mathrm{z}$ & Side & Site & Size $\left(\mathrm{mm}^{3}\right)$ \\
\hline No rivastigmine $(\mathrm{R}-)$ & 42 & -54 & -18 & $\mathrm{R}$ & Fusiform gyrus & 918 \\
& -36 & -74 & -14 & $\mathrm{~L}$ & Fusiform gyrus & 270 \\
& -36 & -51 & -18 & $\mathrm{~L}$ & Fusiform gyrus & 270 \\
Rivastigmine $(\mathrm{R}+)$ & 42 & -51 & -20 & $\mathrm{R}$ & Fusiform gyrus & 1269 \\
& -42 & -51 & -20 & $\mathrm{~L}$ & Fusiform gyrus & 1890 \\
& 42 & -68 & -12 & $\mathrm{R}$ & Fusiform gyrus & 297 \\
$\mathrm{R}+>\mathrm{R}-$ & -42 & -51 & -20 & $\mathrm{~L}$ & Fusiform gyrus & 432 \\
& 42 & -48 & -18 & $\mathrm{R}$ & Fusiform gyrus & 297 \\
\hline
\end{tabular}

$L$, left; R, right.

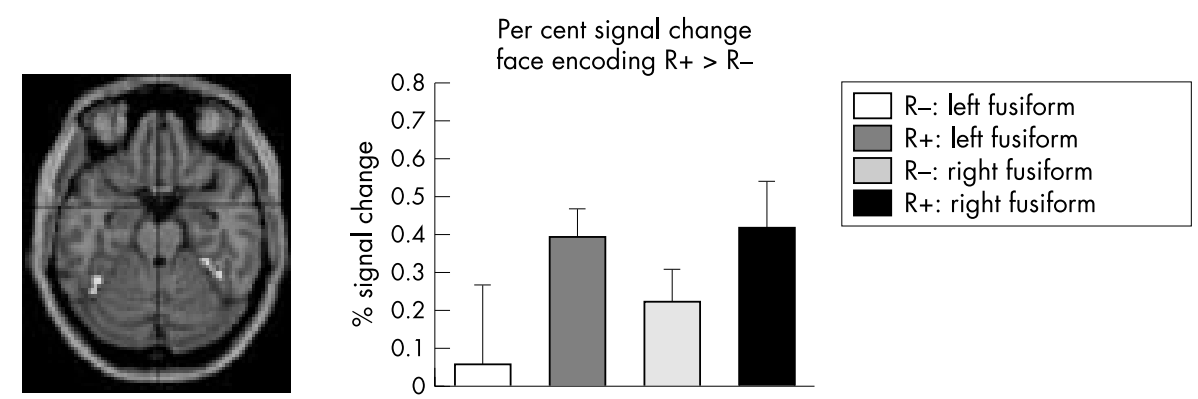

Figure 1 (A) Transverse section in standard coordinate space (left in picture is left in brain) showing an increase in brain activation with rivastigmine treatment during the face encoding task in the left and right fusiform gyri (white areas; $p<0.05$, corrected). (B) Mean relative signal change with standard error between baseline and face encoding without treatment $(R-)$ and during treatment with rivastigmine $(R+)$ of the two local maxima in the left and right fusiform gyri of panel A.

enhanced in $\mathrm{R}+$ ). The local maximum of the contrast $\mathrm{R}+>\mathrm{R}-$ in the left fusiform gyrus did not show a significant effect in the R-arm. Strictly speaking, this treatment effect in the left fusiform gyrus was thus introduced by activation in $\mathrm{R}+$ that was not present in $\mathrm{R}-$. Note, however, that activation in $\mathrm{R}$ - was present near to this voxel (table 2), and that the variance between patients was very high in $\mathrm{R}-$ in that particular voxel (see also fig $1 \mathrm{~B}$ ). The contrast $\mathrm{R}->\mathrm{R}+\mathrm{did}$ not show any effect.

Although our study was designed such that $\mathrm{R}+$ and $\mathrm{R}-$ drug treatment order was counterbalanced, dropouts caused an imbalance of the order in the face encoding task: five patients received rivastigmine in the second session, and two in the first. Therefore, to test whether the observed signal increases in the contrast $\mathrm{R}+>\mathrm{R}$ - in table 2 could also be explained by session order rather than by rivastigmine, we ran an additional analysis testing for interaction between task and session. There were no regions that showed such an interaction.

\section{Working memory}

Five patients performed the working memory task successfully in both sessions. Of these, four received rivastigmine in the second session. As the working memory system was more stressed, the test scores decreased, as expected, while reaction times increased, both in $\mathrm{R}-$ and $\mathrm{R}+$ (table $\mathrm{1}$ ).

The contrast for simple working memory (1-back $>\mathrm{X}$ ) showed brain activation in the R- arm in the inferior and middle frontal gyrus bilaterally, and in the right superior frontal gyrus (table 3 ). In the $\mathrm{R}+$ arm, the bilateral inferior, middle, superior, and medial frontal gyrus, the left inferior parietal lobe, the precentral gyrus bilaterally, and the right postcentral gyrus were activated. The contrast $\mathrm{R}+>\mathrm{R}-$ showed a significant increase in brain activation with rivastigmine in the left middle and left superior frontal gyri (fig 2). These local maxima were not significantly activated in R- (see table 3 and fig 2 ). The contrast $\mathrm{R}->\mathrm{R}+$ (decrease in brain activation with rivastigmine) did not show a significant effect in any area. 
Table 3 Talairach coordinates of all local maxima, including maxima within the same cluster, and sizes of activated clusters during simple working memory (1-back $v \mathrm{X})$ and increased working memory load (2-back $v \mathrm{X})$

\begin{tabular}{|c|c|c|c|c|c|c|c|}
\hline & \multirow[b]{2}{*}{ Cluster No } & \multicolumn{3}{|c|}{ Talairach coordinates } & \multirow[b]{2}{*}{ Side } & \multirow[b]{2}{*}{ Site } & \multirow[b]{2}{*}{ Size $\left(\mathrm{mm}^{3}\right)$} \\
\hline & & $x$ & y & $z$ & & & \\
\hline \multicolumn{8}{|c|}{ Simple working memory } \\
\hline \multirow[t]{6}{*}{ No rivastigmine (R-) } & 1 & -50 & 31 & -12 & L & Inferior frontal gyrus & 1053 \\
\hline & 2 & 48 & 26 & -14 & $\mathrm{R}$ & Inferior frontal gyrus & 1026 \\
\hline & 3 & 48 & 25 & 37 & $\mathrm{R}$ & Middle frontal gyrus & 972 \\
\hline & 4 & 21 & 64 & 5 & $\mathrm{R}$ & Superior frontal gyrus & 945 \\
\hline & 5 & -45 & 33 & 26 & L & Middle frontal gyrus & 675 \\
\hline & 6 & 50 & 32 & 7 & $\mathrm{R}$ & Inferior frontal gyrus & 351 \\
\hline \multirow[t]{14}{*}{ Rivastigmine $(\mathrm{R}+)$} & 1 & 45 & 27 & 7 & $\mathrm{R}$ & Inferior frontal gyrus & 7803 \\
\hline & 2 & -39 & 16 & 32 & L & Middle frontal gyrus & 5346 \\
\hline & 3 & 27 & 34 & 45 & $\mathrm{R}$ & Middle frontal gyrus & 4185 \\
\hline & 4 & -30 & 39 & 31 & L & Superior frontal gyrus & 1404 \\
\hline & 5 & -45 & -9 & 47 & L & Precentral gyrus & 1053 \\
\hline & 6 & -9 & 62 & 16 & L & Superior frontal gyrus & 1026 \\
\hline & 7 & 9 & 55 & -10 & $\mathrm{R}$ & Medial frontal gyrus & 999 \\
\hline & 8 & -39 & -39 & 41 & L & Inferior parietal lobule & 945 \\
\hline & 9 & -48 & 32 & 7 & L & Inferior frontal gyrus & 594 \\
\hline & 10 & 45 & 0 & 8 & $R$ & Precentral gyrus & 486 \\
\hline & 11 & -18 & 58 & -3 & L & Medial frontal gyrus & 459 \\
\hline & 12 & 9 & 39 & 31 & $\mathrm{R}$ & Medial frontal gyrus & 405 \\
\hline & 13 & -3 & 40 & 42 & L & Medial frontal gyrus & 297 \\
\hline & 14 & 65 & -11 & 20 & R & Postcentral gyrus & 297 \\
\hline \multirow[t]{3}{*}{$R_{+}>R_{-}$} & 1 & -9 & 62 & 16 & L & Superior frontal gyrus & 648 \\
\hline & & -15 & 57 & 28 & $\mathrm{~L}$ & Superior frontal gyrus & \\
\hline & $2^{*}$ & -36 & 19 & 29 & $\mathrm{~L}$ & Middle frontal gyrus & 270 \\
\hline \multicolumn{8}{|c|}{ Increased working memory load } \\
\hline \multirow[t]{13}{*}{ No rivastigmine (R-) } & 1 & 48 & 25 & 35 & $\mathrm{R}$ & Middle frontal gyrus & 8721 \\
\hline & & 15 & 65 & 13 & $\mathrm{R}$ & Superior frontal gyrus & \\
\hline & 2 & -56 & 20 & 2 & L & Inferior frontal gyrus & 5832 \\
\hline & 3 & 42 & 20 & -9 & $\mathrm{R}$ & Inferior frontal gyrus & 2079 \\
\hline & 4 & 24 & -70 & 50 & $\mathrm{R}$ & Precuneus & 1215 \\
\hline & 5 & 12 & -67 & 56 & $\mathrm{R}$ & Superior parietal lobule & \\
\hline & 6 & -39 & 46 & -10 & L & Middle frontal gyrus & 1134 \\
\hline & 7 & 45 & 40 & -7 & $\mathrm{R}$ & Middle frontal gyrus & 891 \\
\hline & 8 & 50 & 32 & 7 & $\mathrm{R}$ & Inferior frontal gyrus & 675 \\
\hline & 9 & 6 & -71 & 39 & $\mathrm{R}$ & Precuneus & 432 \\
\hline & 10 & -3 & -53 & 44 & L & Precuneus & 270 \\
\hline & 11 & -30 & -62 & 34 & L & Precuneus & 270 \\
\hline & & -30 & -62 & 50 & L & Superior parietal lobule & \\
\hline \multirow[t]{13}{*}{ Rivastigmine $(\mathrm{R}+)$} & 1 & 15 & 18 & 60 & $\mathrm{R}$ & Superior frontal gyrus & 14931 \\
\hline & 2 & 45 & 23 & -14 & $\mathrm{R}$ & Inferior frontal gyrus & 7776 \\
\hline & 3 & -39 & 39 & 26 & L & Middle frontal gyrus & 2511 \\
\hline & 4 & 39 & -42 & 33 & $\mathrm{R}$ & Supramarginal gyrus & 2160 \\
\hline & 5 & 30 & 46 & -15 & $\mathrm{R}$ & Superior frontal gyrus & 2052 \\
\hline & 6 & -3 & -8 & 64 & L & Medial frontal gyrus & 2052 \\
\hline & 7 & -45 & 46 & -12 & L & Middle frontal gyrus & 1863 \\
\hline & & -33 & 46 & -17 & $\mathrm{~L}$ & Superior frontal gyrus & \\
\hline & 8 & -6 & -52 & 58 & L & Precuneus & 1215 \\
\hline & & -18 & -67 & 56 & L & Superior parietal lobule & \\
\hline & 9 & -42 & -36 & 46 & L & Inferior parietal lobule & 1026 \\
\hline & 10 & -30 & -6 & 61 & L & Middle frontal gyrus & 810 \\
\hline & 11 & -9 & 62 & 19 & L & Superior frontal gyrus & 729 \\
\hline \multirow[t]{6}{*}{$R_{+}>R_{-}$} & $1^{*}$ & -42 & 17 & 41 & L & Middle frontal gyrus & 999 \\
\hline & * & -33 & 20 & 46 & L & Middle frontal gyrus & \\
\hline & * & -53 & 13 & 30 & L & Middle frontal gyrus & \\
\hline & 2 & 45 & 26 & 1 & $\mathrm{R}$ & Inferior frontal gyrus & 729 \\
\hline & 3 & 30 & 43 & -15 & $\mathrm{R}$ & Superior frontal gyrus & 486 \\
\hline & $4^{*}$ & 12 & 17 & 57 & R & Superior frontal gyrus & 405 \\
\hline \multirow[t]{3}{*}{$R->R_{+}$} & 1 & 15 & 65 & 13 & R & Superior frontal gyrus & 972 \\
\hline & & 24 & 59 & 19 & $\mathrm{R}$ & Middle frontal gyrus & \\
\hline & 2 & 36 & 45 & 28 & $\mathrm{R}$ & Middle frontal gyrus & 459 \\
\hline
\end{tabular}

The contrast for increased working memory load (2back $>\mathrm{X}$ ) showed activation in the R- arm bilaterally in the inferior, middle, and superior frontal gyri, precuneus, and the superior parietal lobule (table 3 ). In the $\mathrm{R}+$ arm, the right inferior frontal gyrus, left middle frontal gyrus, left medial frontal gyrus, superior frontal gyrus bilaterally, right supramarginal gyrus, left inferior parietal lobule, left precuneus, and superior parietal lobule were activated. The contrast 

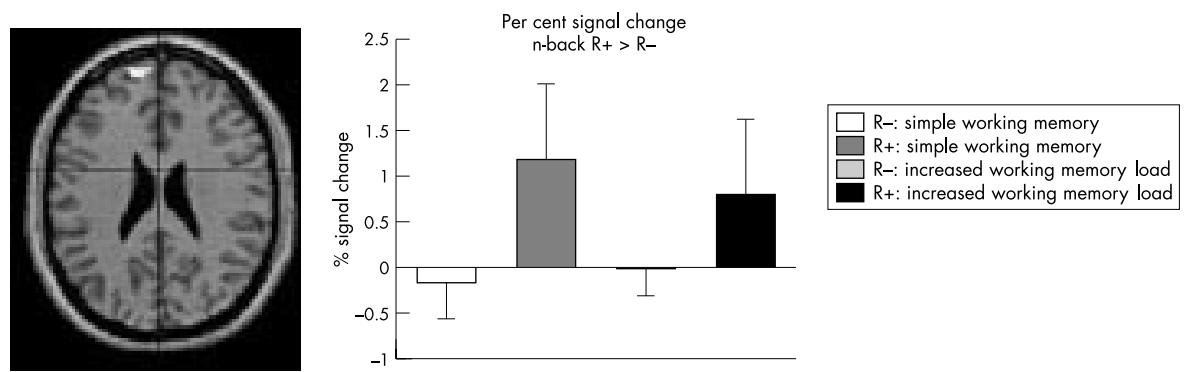

Figure 2 (A) A transverse section showing an increased response with rivastigmine treatment during simple working memory in the left superior frontal gyrus (area in white; $p<0.05$ corrected). (B) Mean relative signal change with standard error during simple and increased working memory load compared with "target $=X^{\prime \prime}$ (baseline) in the local maximum in the left superior frontal gyrus in panel A, showing a treatment effect for simple working memory. Note that there is no activation in R-.
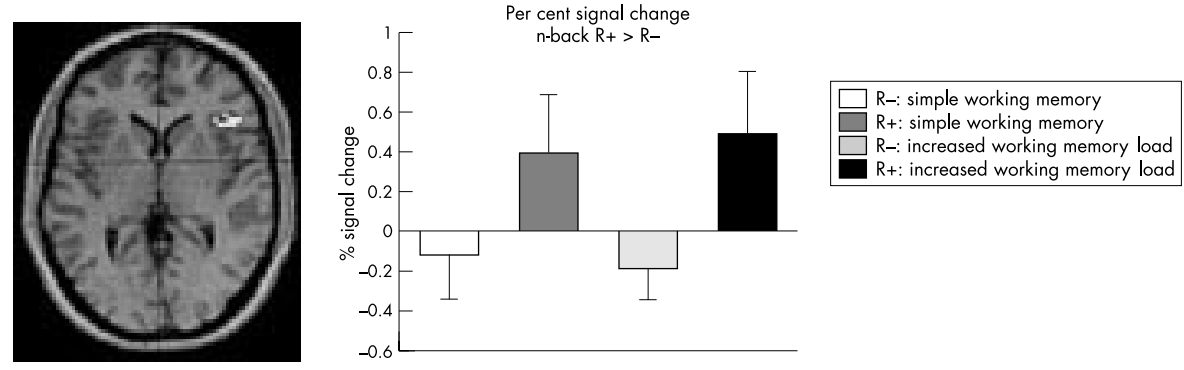

Figure 3 (A) A transverse section showing an increased response with rivastigmine during increased working memory load in the right inferior frontal gyrus (area in white; $\mathrm{p}<0.05$ corrected). (B) Mean relative signal change with standard error during simple and increased working memory load compared with "target $=X$ " (baseline) in the local maximum in the right inferior frontal gyrus in panel $A$.
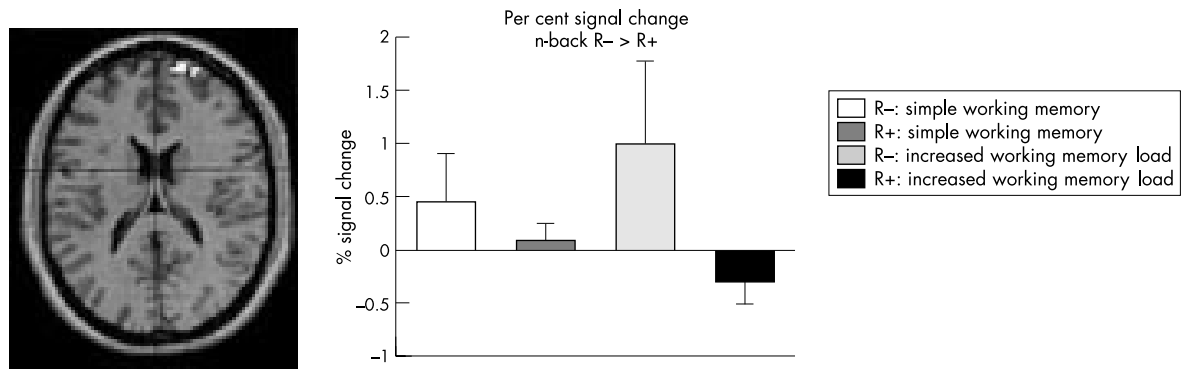

Figure 4 (A) A transverse section showing a decreased response with rivastigmine during increased working memory load in the right middle and superior frontal gyrus (areas in white; $p<0.05$ corrected). (B) Mean relative signal change during simple and increased working memory load compared with "target $=X^{\prime \prime}$ (baseline) in the local maximum in the right superior frontal gyrus in panel $A$.

$\mathrm{R}+>\mathrm{R}-$ showed increased activation in the left middle frontal gyrus, right superior frontal gyrus, and right inferior frontal gyrus (fig 3). None of these local maxima showed a significant effect in R- (see table 3 and fig 4). The contrast R- > R+ showed decreased activation in the right middle and superior frontal gyri (fig 4), local maxima that were not significantly activated in $\mathrm{R}+$ (table 3 , fig 4 ).

For the working memory task, dropouts also resulted in an imbalance in the drug treatment order: four patients received rivastigmine in the second session and only one in the first. As in the face encoding task, we ran additional analyses to test whether the observed signal changes in the contrast R+ $>\mathrm{R}-$ might also be explained by session order rather than by rivastigmine. For simple working memory, there was a significant interaction between test and session in the prefrontal cortex. As a next step, these regional interactions were then included in the mask of the contrast $\mathrm{R}+>\mathrm{R}-$ in order to exclude the possibility of session $\times$ task interactions causing the effects presented in table 3. This showed that the left middle frontal gyrus did not survive this operation, while the left superior frontal gyrus did (table 3 ).

For increased working memory load, there was also a significant task $\times$ session interaction in the prefrontal cortex.
Masking the $\mathrm{R}+>\mathrm{R}-$ contrast with session $\times$ task interaction had the effect that only the right inferior and superior frontal gyri survived the test for the contrast $\mathrm{R}+>\mathrm{R}-$, while the contrast $\mathrm{R}->\mathrm{R}+$ was unchanged.

\section{DISCUSSION}

In this study, we examined the effect of the cholinesterase inhibitor rivastigmine on brain activation using fMRI in patients with Alzheimer's disease. Using a face encoding task, we found increases in brain activation during treatment in the fusiform gyrus bilaterally, and no decreases. Using a working memory task, we found signal increases particularly in the frontal lobe during simple working memory, but there were areas with both increased and decreased activation with increased working memory load. These findings link for the first time the clinically observed increase in cognitive performance after treatment with a cholinesterase inhibitor in other studies ${ }^{1-3}$ to altered brain activation in the current study, suggesting that modified firing rates reflect altered neurotransmitter status in the brain.

During face encoding we observed activation in the ventral occipital cortex, in agreement with other studies of face encoding, ${ }^{78}$ but not in the medial temporal lobe, while other 
studies have shown activation in the medial temporal lobe in healthy subjects, ${ }^{79}$ elderly subjects with memory decline, ${ }^{9}$ and to a lesser extent also patients with Alzheimer's disease. ${ }^{9}$ The lack of activation in the medial temporal lobe in the current study might therefore reflect the Alzheimer process, given the fact that the male/female responses during encoding were correctly performed (presumably reflecting the observed activation in the fusiform gyrus), while face recognition was performed at or just above chance level, probably explaining the lack of medial temporal lobe activation during face encoding. Although rivastigmine has been reported to be hippocampus specific, $^{12}$ we did not find evidence for increased activation in the medial temporal lobe during rivastigmine administration. Interestingly, there was an increase in activation in the fusiform gyrus, which might reflect enhancement of visual face processing on cholinergic enhancement. Such an effect has also been observed by Furey et $a l,{ }^{15}$ who found that the cholinesterase inhibitor physostigmine increased face selective responses in the ventral occipital cortex in healthy subjects. The explanation that the signal increase we observed can be explained by enhanced face processing is further supported by an fMRI study involving drug treatment that induces memory impairment: using a face-name associative encoding paradigm in healthy controls it was found that activation in the fusiform gyrus, and also in the hippocampus and inferior prefrontal cortices, was reduced during pharmacological memory impairment compared with placebo. ${ }^{16}$

As a second test, we applied a parametric non-spatial n-back working memory task. This n-back task has been used to study working memory in healthy subjects. ${ }^{10}{ }^{11}$ To the best of our knowledge, fMRI studies using this task in Alzheimer's disease patients have not been reported. During the working memory task, we found activation in the inferior, middle, and superior frontal gyrus, pre- and postcentral gyri, parietal lobe, and precuneus. This is fairly consistent with other studies that used the n-back task in young healthy subjects, ${ }^{10}{ }^{11}{ }^{17}$ reflecting processes of maintenance and manipulation of items. Although it was not the goal of this study to compare working memory activation between controls and patients with Alzheimer's disease, our data do indicate that activation of the working memory functional system in these Alzheimer patients more closely resembles young healthy subjects than activation of the face encoding memory system. This is in agreement with the well known relation between medial temporal lobe atrophy and Alzheimer's disease, observed in various region of interest based studies, ${ }^{18}$ while such a relation with atrophy in the frontal and parietal cortex has not been reported. Recent work analysing grey matter loss in the whole brain in mild Alzheimer's disease showed that atrophy is most severe in the medial temporal lobe, while parietal and particularly frontal atrophy is much less severe or even absent. ${ }^{19}$

During the simple working memory condition (1-back), we detected signal increases on rivastigmine treatment in the left middle and left superior frontal gyri, but no signal decreases. As in the face encoding task, this can be explained by enhancement of processing in the frontal cortex. When the working memory load is increased and the system is stressed to a higher extent (2-back), we observed both signal increases and signal decreases in the frontal cortex on treatment.

We aimed to study rivastigmine effects in 11 patients. As could be expected in an elderly and demented sample, dropouts occurred, distorting our counterbalanced drug administration scheme ( $\mathrm{R}+$ and $\mathrm{R}-$ ) and session order. Additional statistical tests showed that there was no session $\times$ task interaction for face encoding. Therefore we can reliably conclude that the increased response in the fusiform gyrus is better explained by a drug treatment effect than by session order. For the working memory task, significant task $\times$ session interactions occurred for the contrast $\mathrm{R}+>\mathrm{R}-$ during both simple working memory and increased working memory load. Excluding these regions showed that for the contrast
$\mathrm{R}+>\mathrm{R}-$, during simple working memory the left superior frontal gyrus survived this masking operation, as did the right inferior and superior frontal gyri during increased working memory load. The contrast R- $>\mathrm{R}+$ was unaffected. Therefore, despite the imbalance resulting in regional task $\times$ session interactions, regions could be identified with a signal change better explained by drug effects than by session effects.

Although the behavioural data show that patients were able to perform the tasks and revealed the expected task performance in the working memory task (that is, more errors during increased working memory load), we cannot reliably link the change in brain activation after rivastigmine intake to behavioural data owing to our relatively small sample size and incomplete behavioural data recording.

For both face encoding and working memory, this fMRI study was set up to detect acute effects three hours after the intake of a single dose of rivastigmine, and these were found in the fusiform gyrus and the prefrontal cortex. Studies testing cognition in Alzheimer's disease during rivastigmine treatment have shown significant long term improvement (that is, after 12,18 , and 26 weeks of treatment). ${ }^{21}$ The (expected) accompanying long term effects of rivastigmine on regional brain activation might not be the same as the acute effects in the current study. To address the question of long term effects on brain activation, fMRI studies specifically designed for this purpose should be done.

Owing to patient dropout, our results cannot be generalised to a large Alzheimer's disease population. However, we did find an effect on brain activation in memory related areas in this specific Alzheimer's disease cohort when performing memory tasks. Because of the small sample size, it was not possible to determine whether these functional changes were reflected in significant changes in task performance. Nevertheless, our data suggest enhanced processing (in the fusiform gyrus during face encoding and in the frontal cortex during working memory) in patients with Alzheimer's disease after rivastigmine, linking metabolic intervention, enhanced circuitry, and clinical improvement.

\section{ACKNOWLEDGEMENTS}

This study was financially supported by Stichting Alzheimer and Neuropsychiatry Foundation (Amsterdam), Hersenstichting Nederland (project number H00.17), and Novartis Pharma Nederland.

\section{Authors' affiliations}

S A R B Rombouts, C S van Meel, P Scheltens, Department of Neurology/Alzheimer Centre, Vrije Universiteit Medical Centre, Amsterdam, Netherlands

F Barkhof, Department of Radiology, Vrije Universiteit Medical Centre

Competing interests: none declared.

\section{REFERENCES}

I Jann MW. Rivastigmine, a new-generation cholinesterase inhibitor for the treatment of Alzheimer's disease. Pharmacotherapy 2000;20:1-12.

2 Spencer CM, Noble S. Rivastigmine - a review of its use in Alzheimer's disease. Drug Aging 1998;13:391-411.

3 Forette F, Anand R, Gharabawi G. A phase II study in patients with Alzheimer's disease to assess the preliminary efficacy and maximum tolerated dose of rivastigmine (Exelon®). Eur J Neurol 1999;6:423-9.

4 McKhann G, Drachman D, Folstein M, et al. Clinical diagnosis of Alzheimer's disease: report of the NINCDS-ADRDA Work Group under the auspices of Department of Health and Human Services Task Force on Alzheimer's Disease. Neurology 1984;34:939-44.

5 Morris JC. The clinical dementia rating (CDR): current version and scoring rules. Neurology 1993;43:2412-14.

6 Folstein MF, Folstein SE, McHugh PR. "Mini-mental state". A practical method for grading the cognitive state of patients for the clinician. $J$ Psychiatr Res 1975;12:189-98.

7 Haxby JV, Ungerleider LG, Horwitz B, et al. Face encoding and recognition in the human brain. Proc Acad Natl Sci USA 1996;93:922-7.

8 Kanwisher N, McDermott J, Chun MM. The fusiform face area - a module in human extrastriate cortex specialized for face perception. J Neurosci 1997; 17:4302-11. 
9 Small SA, Perera GM, DeLaPaz R, et al. Differential regional dysfunction of the hippocampal formation among elderly with memory decline and Alzheimer's disease. Ann Neurol 1999;45:466-72.

10 Braver TS, Cohen JD, Nystrom LE, et al. A parametric study of prefrontal cortex involvement in human working memory. Neuroimage 1997:5:49-62.

11 D'Esposito M, Aguirre GK, Zarahn E, et al. Functional MRI studies of spatial and nonspatial working memory. Cognitive Brain Research 1998;7:1-13.

12 Friston KJ, Holmes AP, Worsley KJ, et al. Statistical parametric maps in functional imaging: a general linear approach. Hum Brain Mapping 1995:2:189-210

13 Talairach J, Tournoux P. Co-planar stereotaxic atlas of the human brain Stuttgart: Thieme Verlag, 1988.

14 Genovese CR, Lazar NA, Nichols T. Thresholding of statistical maps in functional neuroimaging using the false discovery rate. Neuroimage 2002;15:870-8

15 Furey ML, Pietrini P, Haxby JV. Cholinergic enhancement and increased selectivity of perceptual processing during working memory. Science 2000;290:2315-19.
16 Sperling R, Greve D, Dale A, et al. Functional MRI detection of pharmacologically induced memory impairment. Proc Acad Natl Sci USA 2002;99:455-60.

17 Cohen JD, Forman SD, Braver TS, et al. Activation of the prefrontal cortex in a nonspatial working memory task with functional MRI. Hum Brain Mapping 1994;1:293-304.

18 Jack CR, Petersen RC, Xu YC, et al. Medial temporal atrophy on MRI in normal aging and very mild Alzheimer's disease. Neurology 1997;49:786-94.

19 Baron JC, Chetelat G, Desgranges B, et al. In vivo mapping of gray matter loss with voxel-based morphometry in mild Alzheimer's disease. Neuroimage $2001 ; 14: 298-309$.

20 Rombouts SARB, Barkhof F, Witter MP, et al. Unbiased whole-brain analysis of gray matter loss in Alzheimer's disease. Neurosci Lett 2000;285:231-3.

21 Rosler M. Efficacy and safety of rivastigmine in patients with Alzheimer's disease: international randomised controlled trial. BM 2001;322:1456.

HISTORICAL NOTE

\section{Amyotrophic lateral sclerosis}

n America, amyotrophic lateral sclerosis (ALS) is often referred to as Lou Gehrig's disease, after the famous baseball player who in 1939 retired because of the disease. ${ }^{1}$ ALS is also known as Charcot's disease (vide infra); but many famous neurologists figure in its history. GBA Duchenne (1806-1875) described a case of the related progressive muscular atrophy (PMA) in 1849, but delayed publishing until $1861 .^{2}$ Aran published the case ${ }^{3}$ and acknowledged:

"I owe a thousand thanks to my friend Duchenne de Boulogne who freely put at my disposal all his material ..."

Luys reported in 1860, the underlying anterior horn cell degeneration; and in the same year Duchenne reported progressive bulbar palsy as "glosso-labial-laryngeal paralysis". ${ }^{4}$ Not until 1883 did Dejerine connect the disorder with ALS. ${ }^{5}$

Charcot's major account in 1865, presented to the Société Médicale des Hôpitaux de Paris, was a woman initially diagnosed as an hysteric who had developed progressive weakness, and increased muscle tone, with contractures of all four limbs. At autopsy, he found no anterior horn cell disease, but he clearly identified lateral column degeneration and sclerosis in the cord ${ }^{6}$ :

"On careful examination of the surface of the spinal cord, on both sides in the lateral areas, there are two brownish-grey streak marks produced by sclerotic changes. These greyish bands begin outside the line of insertion of the posterior roots and their anterior border approaches, but do not include, the entrance area of the anterior roots. They are visible throughout the thoracic region and continue though greatly thinning out, up to the widening point of the cervical cord. Below, they are barely visible in the thoraco-lumbar region. Transverse sections taken at different levels allow one to see that the lateral columns have in their most superficial and posterior regions, a grey, semitransparent appearance, rather gelatinous .... At no point does the diseased tissue penetrate the gray matter which remains unaffected."

In later cases, he found histological changes in both lateral columns and anterior spinal nerve roots which enabled him to identify both the upper and lower motor neurone motor pathways. He summarised the clinical accompaniments

"Paresis without anaesthesia, of the upper extremities, accompanied by rapid emaciation of the muscular masses and often preceded by numbness and formication. Spasmodic rigidity seizes, at a given period, on the paralysed and wasted muscles and determines permanent deformations by contracture. The lower extremities are invaded in their turn . . . . A third period . . . the appearance of bulbar symptoms".

Charcot then demonstrated lesions in the brain stem associated with weakness of the muscles of the face, mouth, and tongue. In 1871-2, his student Albert Gombault (1844-1904) showed symmetrical sclerosis of the lateral columns and of the bulbar pyramids. ${ }^{8}$ In Charcot's laboratory, Aleksei Yakovlevich Kozhevnikov (1836-1902) importantly showed that the spinal degeneration could be traced to the motor cortex. Charcot commented that many investigators had previously tried without success to correlate a primary lesion of the grey matter of the medulla with the clinical signs known as glosso-labial-laryngeal paralysis.

The term amyotrophic lateral sclerosis was first used in 1874 in Charcot's two lectures. ${ }^{7}$ A year later Erb reported primary lateral sclerosis - that was to cause continuing nosological confusion. It was Gowers who combined PMA, progressive bulbar palsy, and ALS as

"Motor Neurone disease" in 1892; in the US, the customary name is "Combined System disease".

Recent experiments in transgenic mice suggest that in ALS there may be cytoskeletal abnormalities including abnormal inclusions containing neurofilaments and/or peripherin, reduced mRNA levels for the NF light protein, and mutations in the NF heavy gene."

J M S Pearce

304 Beverley Road, Anlaby, Hull HU10 7BG, UK; jmspearce@freenet.co.uk

\section{References}

1 Mitsumoto H, Chad D, Pioro E. History, terminology, and classification of ALS. In: Amyotrophic Lateral Sclerosis. Philadelphia: Oxford University Press, 1998:4-5.

2 Duchenne GBA. La paralysie atrophique musculaire progressive. In L'electricisation localisée (2nd edn). 1861;437-547. In: GV Poore (translated). Selections from the clinical works of Dr Duchenne (de Boulogne). London: The New Sydenham Society, 1883:42-87.

3 Aran FA. Recherches sur une maladie non encore décrite du système musculaire (Atrophie musculaire progressive). Archives Génerales de Médicine 1850;4:4-35.

4 Duchenne GBA. Paralysie musculaire progressive de la langue, du voile du palais et des levres; affection non encore decrite. Arch gén Méd 1860;5:283-96, 431-45.

5 Dejerine JJ. Arch Physiol Paris 1883;2:180-227.

6 Charcot J-M, Sclérose des cordons latéraux de la moelle épinière chez une femme hystérique atteinte de contracture permanente des quatre membres. Bull de la Société Méd des Hôpit de Paris. 1865:24-35.

7 Charcot J-M. On Amyotrophic lateral sclerosis. Symptomatology. 1881 In G Sigerson (translated). Lectures on Diseases of the Nervous System. London: New Sydenham Society, 1881:192-204, 205-32. See also Gaz Hôp Paris 1865;38:93-5.

8 Gombault A. Sclérose symétrique des cordon latéraux le bulbe. Arch Physiol Norm et Pathol 1871-2;4:509-18.

9 Julien JP, Beaulieu JM. Cytoskeletal abnormalities in amyotrophic lateral sclerosis: beneficial or detrimental effects? J Neurol Sci 2000;180:7-14. 